\title{
The use of hydraulic models to examine the performance of storm-sewage overflows
}

\author{
A. R. HALLIWELl \& A. J. SAUL
}

\section{Mr H. R. Oakley. Watson Hawksley}

I appreciate the careful approach to model design but critical factors appear to be the very low efficiencies at low $q / Q$ ratios when the particle terminal velocity is small, and the effect of particle size. There is little published information on size and terminal velocity ranges of particles in storm sewage and without this information model studies would be premature. If the majority of particles fell in the critical band, model studies would not in any event be of much significance.

\section{Professor D. I. H. Barr, University of Strathclyde}

Storm-sewage overflow chambers of the Sharpe and Kirkbride configuration ${ }^{1}$ share with a number of similar circumstances the property of being basically Froudian in nature. This factor dominates the overall throughflow characteristics when a conventional free surface model is operated. However, the free surface has two distinct parts. The first part is virtually horizontal and extends over the major portion of the chamber, which portion is of greatest interest in the matter of the separating capacity of the chamber. The second part relates to the actual overflow circumstance. As the Authors show, the achievement of Froudian similarity in a scale free surface model necessarily means that the Reynolds number of the model is much smaller than the Reynolds number of the prototype. It may be so small as to cause significant scale effect.

42. Suppose that the model were modified by the addition of a sealed cover approximating to the predetermined free surface level over the major parts of the chamber, but with appropriate local shaping to impose the flow pattern of the actual overflow at the throughflow of greatest interest. Then the Reynolds number could be much increased by increasing the flow velocity to the limit achievable with laboratory scale pressurization. Certainly, a pressure head of several meters could be envisaged. It might be that the tendency for a change in velocity distribution at the 'free surface' as between pressurized model and prototype should then be compensated for by lifting the cover level marginally and by ensuring that the cover was of the smoothest material, while the other surfaces of the tank were slightly roughened.

43. With this situation, the system could be run with quite high Reynolds numbers, probably not reaching prototype values, but certainly above the limits

Paper published: Proc. Instn Civ. Engrs, Part 2, 1980, 69, June, 245-259. 
where significant changes in the main subsurface flow patterns might be expected because of viscous scale effect. Also, the average velocity of inflow could be varied to suit the settling velocity of commercially available material of close to neutral buoyancy, which could then be used to simulate the particles of the prototype situation. A combination of different materials might be used, to move towards the prototype situation. If the model particles no longer had to be individually graded, greater quantities could be used. With appropria te measures to overcome the difficulty of entry of particles to a pressurized system, these could then be collected in sufficient quantity to make comparative assessments straightforward. Again, care would have to be taken to allow good settling after the high velocity section of flow in the actual model. If a range of materials were used, the mixed collection of materials which had been carried in the overflow would have to be sorted, possibly aided by a resettling process.

44. What do the Authors think are the possible advantages or disadvantages of this approach? It is a difficult approach to recommend once a project is in hand but while substantiation is still lacking. I have been in the same position as the Authors and have followed the same basic (Froudian) option as they did. I believe it would be helpful if a programme of comparative testing of Froudian and of pressurized models of a chosen prototype could be undertaken to prove or disprove the feasibility of the suggested approach. Comparisons might be made with valuable results which have already been obtained by the Authors. The benefits which might accrue for future studies would arise as much from the capacity to use more readily available and less finely selected particle materials as from the changes in flow patterns obtaining with increase in Reynolds number.

\section{Mr R. Burrows and Dr K. H. M. Ali, University of Liverpool}

We support the conclusions made by the Authors and would like to extend the comments made in $\$ 34$ and $\$ 37$ by suggesting that hydraulic model testing of new overflow designs should normally be conducted with a conventional 'standard' structure of the same scale, tested in parallel. Use of a range of different types of particulate should produce a suitable data base for assessment of the acceptability of the new design. Such a procedure eliminates the considerable uncertainty associated with formulating comparisons with previous work to account properly for any differences in the particles tested.

46. The definition of efficiency ("percentage of particles which do not pass over the overflow') given in the Paper implies that the measuring period of the particle retention tests (the time after particle entry in to the chamber when overflow losses are counted) is of no importance. This implies that the particle's destination, either retention or overflow, is determined from its motion directly on entry only. In tests we conducted recently, using injections of particles in large numbers $(>300)$, it was evident that the overflow of particles, in particular the buoyant ones, also results from re-entrainment in to the main flow paths following initial separation at entry into 'quiescent' zones in the chambers. This loss mechanism was most significant for the high flow rates tested (roughly equivalent to the two-year flow) for which the particle terminal velocity to inflow velocity ratio $W / U_{0}$ was of the order of $0 \cdot 15$. We were unable to ascertain whether this effect retained its importance for particles of lower rise velocity when subjected to a lower range of flow rates as no appropriate particulate was available. Judging from comments made by Sharpe and Kirkbride ${ }^{1}$ and Frederick and Markland ${ }^{2}$ concerning their choice of two-minute 
measuring periods because periods of ten minutes made little difference to the results, from tests using only small batches of particles (10-20), it would appear that this may not be the case. Were such effects observed by the Authors and at what time after entry of the particle, in the single particle tests, was the decision on its destination made?

47. Our study, conducted for Liverpool City Engineer's Department, involved the comparison of a non-standard 'shaft' overflow arrangement, devised for a deep interceptor sewer application (Fig. 7) with a standard Sharpe-Kirkbride stilling pond. Models of the same scale were constructed and tested using nodules of various polymers as particulate. Variations in size and shape from particle to particle necessitated the use of injection in large numbers to minimize the sampling errors in efficiency estimates from run to run, resulting from differences in the distribution of particle characteristics from batch to batch. Using the procedure efficiency measurements were reasonably consistent from run to run, normally differing by less than $\pm 15 \%$. However, in exceptional cases associated with high flow rates and low efficiencies, deviations of up to $40 \%$ were observed for the stilling pond structure.
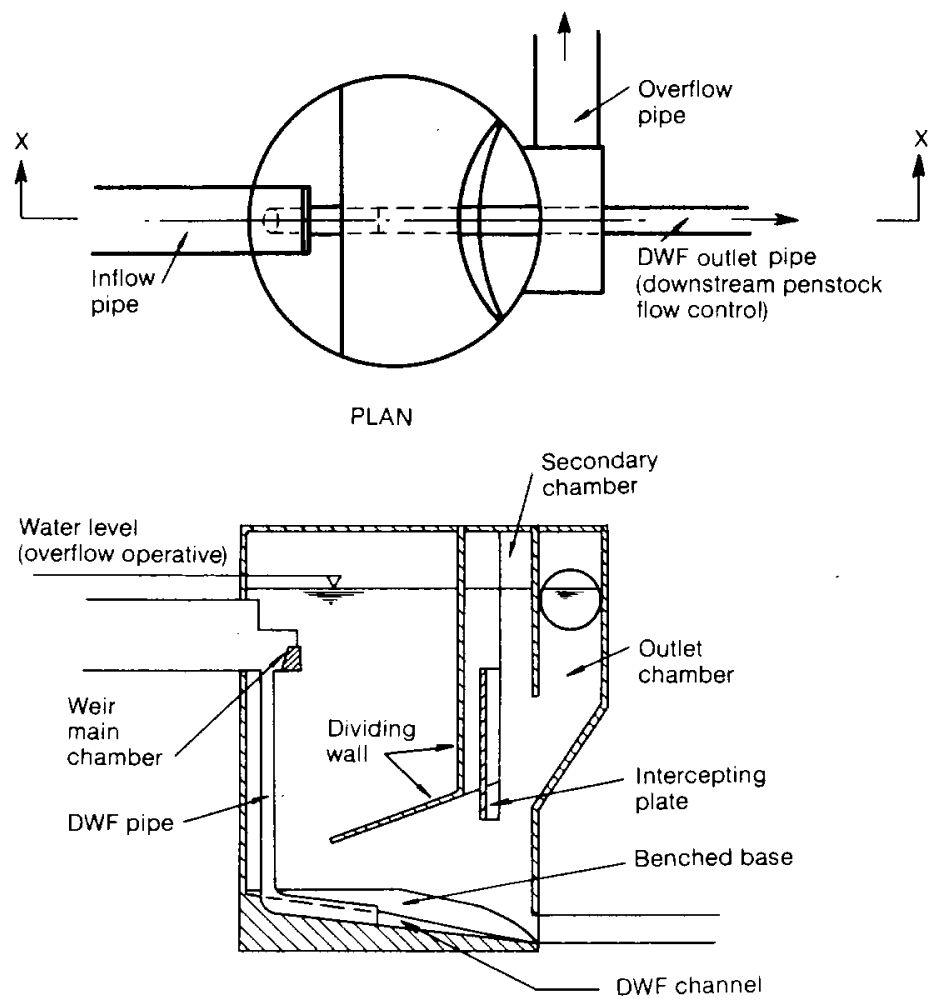

SECTION $X X$

Fig. 7. Shaft overflow 
48. Although the single particle injection method is clearly superior in reliability to the above procedure it would probably be impractical for most model testing studies where time available is often severely restricted. For long-term studies it may well be more cost effective to have particulate produced in large numbers to fine tolerances to effect experimental time savings without detriment to the quality of the results.

49. Concerning the presence of two mechanisms of overflow loss - the direct loss following entry and the loss following re-entrainment from the surface or bedit is possible that relative efficiency results obtained in comparison tests of one chamber against another may be dependent on the measurement period of the tests, under high flow rates at least. As an illustration, in our comparison tests the nonstandard shaft arrangement, with a more effective barrier and longer flow path between inlet and outlet than the stilling pond, minimized direct losses of buoyant particulate but showed a greater rate of re-entrainment loss, due to its smaller surface area and restricted surface quiescent zones. Consequently, the relative performance of the shaft to the stilling pond deteriorates with increase in the measurement period. In these circumstances the relative performance of different chambers under high flow conditions (and possibly under a lower range of flows with more neutrally buoyant particles if the re-entrainment loss mechanism is still found to be significant) cannot be ascertained from steady state tests alone because account must be made of temporal effects reflecting continuous particle entry and the duration of the flow condition.

50. The shaft arrangement showed similar retention characteristics to the stilling pond for buoyant particles, based on tests conducted with a four-minute measuring period, but was found to offer considerable improvement in its retention of non-buoyant particulate.

\section{Professor Halliwell and Dr Saul}

Mr Oakley rightly points out that the critical factor of practical significance with all the overflow chambers tested is the very low efficiencies obtained at low $q / Q$ ratios when the terminal velocity is small. By and large, we agree with his comments concerning the importance of having information about the sewage particulate. Indeed in reference 7 we emphasized the need for information concerning the hydraulic characteristics of the particulate matter in sewage. Subsequently, work has been carried out at Heriot-Watt University to study the terminal velocities of particulate matter in samples taken from two sites near Edinburgh. About 100 sewage samples taken over 18 months were analysed. ${ }^{14}$ The results showed that although a large proportion of the particulate has very low terminal velocities there is a sufficient amount of particulate having terminal velocities greater than $70 \mathrm{~mm} / \mathrm{s}$ to justify the careful design and construction of storm-sewage overflow chambers.

52. Clearly, such information about the particulate is necessary if objective comparisons are to be made properly between different overflow chambers. However, we do not agree that "without this information model studies would be premature'. The design and construction of storm-sewage chambers continues without such information and one of the objectives of such devices is to reduce to minimal levels the pollution of the natural waterways into which the storm-sewage water is discharged. Model tests, even without information about the sewage, will at least give an indication of the general performance of a design proposal and may well show that some designs should be rejected and others improved. 
53. If information about the hydraulic characteristics of the particulate in a storm sewer is available for a particular site it is possible to determine an overall efficiency value for different chambers, should they be used at the site in question. This can be obtained by multiplying the proportion of the particulate having a given terminal velocity by the efficiency, determined from model tests, for that particulate; summing for the entire range of terminal velocities occurring gives an overall efficiency value for a given storm flow. This overall efficiency for a stormsewage chamber may be a useful, and easily comparable, measure of its performance. ${ }^{7,8}$

54. Professor Barr draws attention to the fact that significant scale effects may arise from the lack of dynamic similarity associated with the comparatively low Reynolds' numbers in the model. He suggests a most interesting approach which might reduce any such scale effects. Two major difficulties of operating the technique are mentioned by Professor Barr: the difficulty of achieving the pressurization of the chamber without disturbing unduly the flow near the free surface and overflow weir, and the difficulties associated with the entry of particles to a pressurized system. We think that the use of a sealed cover, even when constructed to include appropriate local shaping and with as smooth a surface as possible, is likely to alter significantly the flow pattern in certain areas and especially near the overflow weir. The suggestion that the cover be lifted marginally is a good one, but it is difficult to see how this could be done without further complications at the overflow weir. It may be that the use of such a model will cause more scale effects, especially near the free surface, than it removes. This possibility causes us to suggest that it may be best to operate two models-the basic Froude-type model and the pressurized one - in parallel and thereby enable direct (simultaneous) comparisons to be made.

55. The major advantages of the approach are almost certainly associated with the high inlet pipe velocities in the model which will, in turn, enable a much wider range of particles to be used to represent the almost neutrally buoyant particles. It seems likely that the model velocities with such an arrangement will be about the same as (maybe bigger than) those occurring in the prototype and therefore, as Professor Barr suggests, commercially available material could be used to simulate the particulate, and it may not be necessary to select the particles so carefully. This would make the testing much less laborious and time-consuming. Overall, it would be most interesting and worthwhile to see comparisons between the two types of model for at least one storm-sewage overflow chamber; the difficulties, although formidable, are not insurmountable and the potential advantages are considerable.

56. In reply to Mr Burrows and Dr Ali, in defining the efficiency of the system as the percentage of particles which do not pass over the overflow (\$19) we did not intend to convey the idea that the measuring period for each test is of no importance. We have given this matter a good deal of attention. ${ }^{7,8}$ For any particles which are retained in the chamber (often behind a baffle) until the storm has subsided it is clear that the measured efficiency can depend on the time allowed for the beads or particles to pass through the system before the efficiency is calculated. This applies especially (if not solely) to those particles which have a relatively large rising terminal velocity. An almost arbitrary decision has to be made about the time which will be allowed after inserting the beads in the inlet pipe before noting where they are retained, for the purpose of calculating the efficiency. We decided to use a period of 5 minutes for all the tests; with the scale used this corresponded to about 
12 minutes in the prototype, which is reasonable, but if anything rather too small to represent an average storm. Fortunately, most particles passed through the chambers in less than three minutes and therefore these efficiency measurements would not have been changed if the period allowed was increased.

57. The suggestion that model tests for any new overflow design should be conducted with a standard system, of the same scale, tested in parallel is excellent. This would go a long way towards giving comparative data which are both quantitative and repeatable. However, we do not think that this would succeed unless great care were also taken to ensure that the efficiency measurements are accurately determined, which in turn requires great care in choosing the particulate. Small differences in efficiency are usually important and we have found that the required accuracy can be obtained only by using particles which comprehensively cover the full range of terminal velocities and by taking great care to obtain repeatable results. Although the single particle injection method is timeconsuming, it is not necessarily very expensive (compared with the total cost of modelling) and enables efficiency measurements to be obtained accurately so that reliable comparisons can be made. If it is possible to produce particles in large numbers to fine tolerances and thereby effect experimental time savings without detriment to the quality of the results, then the method used by Mr Burrows and Dr Ali is clearly the preferred approach. However, our experience is that at the present time it is not possible to obtain such particles and the use of commercially available particles usually leads to large variations in the measured efficiency values. The variations in measured efficiency of up to $40 \%$ for the non-standard shaft overflow arrangement indicate that the variations in particle characteristics did not fall within acceptable limits. Such variations in measurement make it impossible to make proper comparisons between two systems.

58. The shaft overflow arrangement tested by Mr Burrows and Dr Ali demonstrates how local circumstances and ground topography place important constraints on any design proposals. In many situations the inlet and outlet inverts are at almost the same level, whereas for the Liverpool situation the difference in level appears from Fig. 7 to be considerable. It would seem that the success of that design depends to some extent on its ability to retain the floating particulate in the chamber until the storm subsides. The real efficiency of such a chamber is obviously influenced by the duration of the storm and the shape of the flood hydrograph. The Paper expresses our view that the inclusion of any unsteady aspects greatly complicates the testing and the rewards do not seem to justify the effort. If information were available about the distribution with time during the storm of sewage particulate entering the chamber, then it is possible that suitable model tests would be useful. The steady-state measurements of efficiency obtained using models are valuable because they provide simple quantitative comparisons between chambers. That part of the performance of any chamber which is associated with the timevarying flow conditions is probably best assessed in the prototype itself.

\section{Reference}

14. Scottish Development DePartment. Settling velocities of particulate matter in sewage. Scottish Development Department, Edinburgh, 1980, Report ARD 6. 